\title{
Oficinas educativas sobre HIV/Aids: uma proposta de intervenção para idosos
}

\author{
Educative workshops on HIV/Aids: proposing interventions for elderly
}

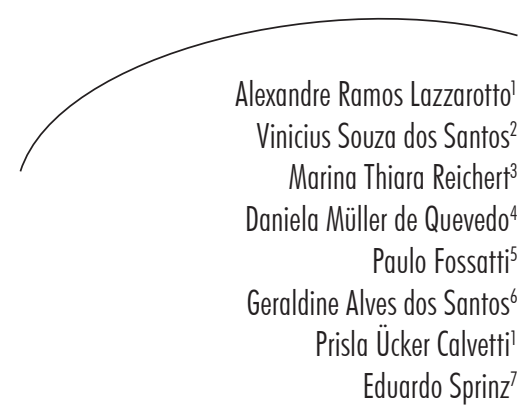

\section{Resumo}

O objetivo do estudo foi avaliar a eficácia de uma oficina educativa sobre HIV/ aids em um grupo de idosos. A população incluiu 471 indivíduos entre 60 e 91 anos composta principalmente por mulheres $(83,1 \%)$. Procedeu-se a aplicação e reaplicação de um questionário organizado em cinco domínios, antes e após as oficinas. O teste de McNemar foi utilizado para comparar as respostas pré e pós-oficina $(\mathrm{p}<0,05)$ no programa estatístico STATA 12. Dentre os domínios, a maior variação $(202,72 \%)$ ocorreu no domínio "conceito" sobre a fase assintomática da infecção. No domínio "transmissão", a variação foi de $168,53 \%$ para a transmissão do HIV por picada de mosquito. No domínio "prevenção", na questão sobre existência do preservativo feminino, a variação foi de 44\%. O domínio "vulnerabilidade" indicou 34,93\% na questão da aids relacionada a grupos específicos. No domínio "tratamento", a abordagem da cura para a aids obteve $50,85 \%$ de variação. A realização das oficinas demonstrou ser uma intervenção eficaz nos domínios "conceito", "transmissão", "prevenção", "vulnerabilidade" e "tratamento" nos idosos participantes da pesquisa.

\section{Abstract}

This study aimed to evaluate the effectiveness of educational workshops instructing about HIV/Aids in a group of elderly. The population included 471 individuals between 60 and 91 years old, mostly women (83.1\%). A questionnaire comprising five domains was answered by participants before and after taking the educational workshops.

\footnotetext{
Programa de Pós-graduação em Saúde e Desenvolvimento Humano. Centro Universitário La Salle. Canoas, RS, Brasil.

2 Curso de Enfermagem. Centro Universitário La Salle. Canoas, RS, Brasil.

3 Curso de Quiropraxia, Instituto de Ciências da Saúde. Universidade Feevale. Novo Hamburgo, RS, Brasil.

4 Centro de Pesquisa e Planejamento. Universidade Feevale. Novo Hamburgo, RS, Brasil.

5 Programa de Pós-graduação em Educação. Centro Universitário La Salle. Canoas, RS, Brasil.

6 Curso de Psicologia. Universidade Feevale. Novo Hamburgo, RS, Brasil.

7 Serviço de Medicina Interna, Unidade de Doenças Infecciosas. Hospital de Clínicas de Porto Alegre. Porto Alegre, RS, Brasil.
}

Correspondência / Correspondence

Alexandre Ramos Lazzarotto

E-mail: alazzar@terra.com.br
Palavras-chave: Síndrome de Imunodeficiência Adquirida. HIV. Conferências de Consenso como Assunto. Idoso. Oficinas. 
McNemar test was used to assess differences between pre-and post-workshop, through thr software STATA 12 ( $\mathrm{p}<0.05)$. The greatest variation $(202.72 \%)$ was observed in the "concept" domain related to the asymptomatic infection phase. Regarding "HIV transmission", the variation was $168.53 \%$. The domain "prevention" had variation of $44 \%$ concerning the existence of female condom. The "vulnerability" domain inquired about Aids association in specific groups, and a difference of $34.93 \%$ was observed. In the "treatment" domain, when inquiring about Aids cure, 50.85\% variation was observed. The educational workshops were effective for the domains "concept", "transmission", "prevention", "vulnerability" and "treatment" in a group of elderly.
Key words: Acquired

Immunodeficiency

Syndrome. HIV. Consensus

Development Conferences as Topic. Elderly. Workshops.

\section{INTRODUÇÃO}

A aids é o estágio final da infecção pelo HIV, caracterizada por uma doença oportunista e ou contagem de linfócitos T CD4+ abaixo de 350 células $/ \mathrm{ml}^{1}$ As principais tendências da epidemia são a pauperização e a interiorização, principalmente pela dificuldade de acesso à informação e aos serviços de saúde entre a população marginalizada e, ainda, pela insatisfatória qualidade dos serviços de vigilância oferecidos nas pequenas localidades. ${ }^{2}$

Os casos de aids na faixa etária a partir dos 60 anos notificados no Brasil até junho de 2012 totalizaram 18.712, sendo que a subnotificação alerta para um número que pode ser bem maior. ${ }^{3}$ No Brasil, de 1980 a 2012, foram notificados 656.701 casos. $^{3}$ A maior taxa de incidência no Brasil em 2011 situou-se na Região Sul, com 30,9 casos por 100 mil habitantes. ${ }^{3}$ No Rio Grande do Sul notificou-se, até junho de 2012, o total de 65.852 casos, caracterizando o mesmo perfil da infecção que o país: aumento de adultos infectados com mais de 40 anos de idade e diminuição proporcional entre adultos com idade inferior a 29 anos. $^{3}$ Salienta-se que o uso da terapia antirretroviral, a partir da introdução dos inibidores da protease em 1996, foi um fator determinante para garantir o envelhecimento dos adultos com HIV/aids.

O desenvolvimento de ações de promoção à saúde, prevenção de doenças e equidade no acesso aos cuidados de saúde servem como bases para um processo de envelhecimento saudável. ${ }^{4}$ As ações devem ser dirigidas aos indivíduos, grupos específicos e à população em geral, considerando-se os aspectos relacionados à vulnerabilidade, influenciada por fatores individuais, sociais, econômicos, institucionais e culturais. $^{2,5-7}$

O estudo realizado por Lazzarotto et al. ${ }^{8}$ constatou lacunas no conhecimento sobre HIV/Aids em participantes de grupos de convivência da terceira idade, evidenciandose, desta forma, a necessidade de implantação de programas de educação para a saúde. Citase, como exemplo, o desconhecimento da fase assintomática da infecção, a questão que mais gerou dúvida no estudo, com 49,4\% de erro nos participantes idosos.

O estudo de Souza et al., ${ }^{9}$ que também avaliou o nível de conhecimento em um grupo de idosos no Rio Grande do Sul, corrobora a necessidade do desenvolvimento de programas sobre os principais aspectos da doença que, como decorrência, possam diminuir a incidência de casos de aids e do preconceito com os portadores do HIV. Devido à carência de trabalhos que avaliem as intervenções sobre HIV/Aids em indivíduos da terceira idade, desenvolveu-se este estudo, com o objetivo de avaliar a eficácia de uma oficina educativa sobre o HIV/Aids em um grupo de idosos.

\section{MÉTODO}

O delineamento foi por séries temporais. A população participante do estudo foi composta por 471 integrantes da terceira idade de 30 grupos de convivência das regiões do Vale do Rio dos Sinos, do Paranhana-Encosta da Serra 
e das Hortênsias-RS, Brasil. O período de coleta dos dados ocorreu entre abril de 2007 e junho de 2011.

$\mathrm{O}$ contato inicial foi realizado com o coordenador de cada grupo, explicando-lhe todas as etapas da pesquisa, dirimindo as dúvidas e solicitando autorização para coletar os dados. A pesquisa teve autorização expressa dos coordenadores dos 30 grupos de convivência oriundos de clubes, entidades religiosas e organizações da sociedade civil.

A seleção dos participantes foi realizada de acordo com os seguintes critérios: de inclusão indivíduos de ambos os sexos, a partir dos 60 anos de idade, integrantes dos grupos de convivência da terceira idade; e de exclusão - indivíduos que já tivessem assistido a alguma palestra ou oficina abordando infecções sexualmente transmissíveis ou que não participassem de um dos encontros do estudo.

A coleta de dados foi operacionalizada nas seguintes etapas: a) apresentação do grupo de pesquisadores e da proposta de investigação aos participantes; b) elucidação das dúvidas; c) convite e assinatura do Termo de Consentimento Livre e Esclarecido; d) aplicação do questionário; e) realização da oficina; f) reaplicação do questionário. Reiterou-se que os integrantes dos grupos que não quisessem preencher os questionários poderiam assistir a oficina e entregar esses instrumentos sem nenhum preenchimento aos pesquisadores.

O questionário HIV para terceira idade $(\mathrm{QHIV} 3 \mathrm{I})^{10}$ contemplou as características gerais dos participantes (idade, sexo, religião, presença de companheiro(a), escolaridade e renda mensal), o uso de preservativos, a realização de testagem para HIV, o conhecimento de portador do vírus pelo participante e 13 questões organizadas nos domínios "conceito", "transmissão", "prevenção", "vulnerabilidade" e "tratamento", as quais apresentam como resposta as alternativas "verdadeiro", "falso" e "não sei". O QHIV3I foi elaborado com base na literatura científica, submetido à avaliação cega interjuízes, aplicado por procedimentos de teste e reteste em grupos de convivência da terceira idade e teve a fidedignidade avaliada pela utilização do coeficiente Kappa.

Em todos os grupos, as oficinas foram desenvolvidas em dois encontros. No primeiro, foi apresentada a pesquisa com o respectivo convite, assinatura do Termo de Consentimento Livre e Esclarecido e respondido o QHIV3I ${ }^{10}$ (primeira coleta dos dados). Com base nas lacunas no conhecimento dos participantes, identificadas na aplicação do questionário, foi estruturada a ênfase da oficina para o grupo, com recurso multimídia e distribuição de fôlderes, cartazes e preservativos masculinos, com duração entre 40 e 50 minutos. A dinâmica utilizada nas oficinas foi pautada pelo acolhimento e sensibilização dos participantes sobre a temática HIV/ Aids; procedimentos expositivos dialogados (de acordo com as lacunas); distribuição e discussão do conteúdo do fôlder e do cartaz; explicação sobre o uso dos preservativos masculino e feminino; distribuição e manuseio do preservativo masculino e um período final para outros questionamentos sobre HIV/Aids que fossem importantes para os participantes. $\mathrm{O}$ preservativo feminino foi demonstrado, porém, não foi distribuído, pois, em decorrência do seu custo elevado, o Ministério da Saúde não o disponibiliza amplamente nos serviços em HIV/ Aids. Em algumas intervenções, houve o auxilio de um intérprete que traduziu do português para o dialeto alemão falado no grupo pesquisado.

Após o término da oficina, realizou-se novamente a aplicação do questionário (segunda coleta dos dados). Os indivíduos que não participaram de um dos encontros não foram incluídos no estudo (10\% a $20 \%$ da totalidade de cada grupo), mas foram convidados a participar da oficina aqueles que não compareceram no primeiro encontro.

O tratamento estatístico foi realizado por frequência e porcentagem para descrever as características gerais dos participantes, o perfil de vulnerabilidade e as maiores variações obtidas em cada domínio. O teste de McNemar foi utilizado para comparar as respostas pré e pós-oficina $(p<0,05)$ no programa estatístico STATA 12. 


\section{RESULTADOS}

Os participantes situaram-se na faixa etária entre 60 e 91 anos (média de 69,41), com 83,1\% (391 participantes) do sexo feminino. Quase a metade dos participantes $(49 \%)$ possuía uma renda mensal de até um salário mínimo e escolaridade de quatro a sete anos de estudo (45\%). A presença de companheiro(a) (51\%) e a religião católica (61\%) foram predominantes entre os participantes (tabela 1). Na questão sobre o conhecimento de alguma pessoa portadora do HIV, houve a prevalência de $23 \%$ (109 participantes).

Tabela 1 - Características gerais dos participantes $(n=471)$. Vale do Rio dos Sinos, Paranhana-Encosta da Serra e Hortênsias, RS, 2007-2011.

\begin{tabular}{|c|c|c|}
\hline Variáveis & $\mathrm{n}$ & $\%$ \\
\hline \multicolumn{3}{|l|}{ Sexo } \\
\hline Masculino & 80 & 17 \\
\hline Feminino & 391 & 83 \\
\hline \multicolumn{3}{|l|}{ Idade } \\
\hline Mínima & 60 & \\
\hline Máxima & 91 & \\
\hline Média e desvio-padrão & $69,41 \pm 8$ & \\
\hline \multicolumn{3}{|l|}{ Religião $^{1}$} \\
\hline Católica & 285 & 61 \\
\hline Evangélica & 136 & 29 \\
\hline Outras & 7 & 1,8 \\
\hline \multicolumn{3}{|l|}{ Companheiro(a) ${ }^{2}$} \\
\hline Sim & 242 & 51 \\
\hline Não & 222 & 47 \\
\hline \multicolumn{3}{|c|}{ Renda mensal (em salários mínimos) ${ }^{3}$} \\
\hline Até 1 salário & 230 & 49 \\
\hline 1 a 3 salários & 187 & 40 \\
\hline 4 a 6 salários & 32 & 7 \\
\hline 7 a 8 salários & 6 & 1 \\
\hline 9 a 10 salários & 1 & 0 \\
\hline Mais de 10 salários & 3 & 1 \\
\hline \multicolumn{3}{|l|}{ Escolaridade (em anos de estudo) ${ }^{4}$} \\
\hline Nenhuma & 36 & 8 \\
\hline 1 a 3 anos & 169 & 36 \\
\hline 4 a 7 anos & 213 & 45 \\
\hline 8 a 11 anos & 27 & 6 \\
\hline 12 ou mais anos & 22 & 5 \\
\hline
\end{tabular}

${ }^{1}$ Não informado $9 \%(43) ;{ }^{2}$ não informado $1 \%(3)$, nulo $1 \%(4) ;{ }^{3}$ não tem renda $1 \%(5)$, não informado $1 \%(5)$, nulo $0 \%(2) ;{ }^{4}$ não informado $1 \%(3)$, nulo $0 \%(1)$. 
Considerando o perfil de vulnerabilidade dos participantes, a maioria ( $84 \%$ ) não utilizava preservativo e não havia realizado o teste antiHIV (91\%) (tabela 2).
Na tabela 3, estão descritas as comparações das respostas obtidas antes e após as oficinas.

Tabela 2 - Perfil de vulnerabilidade dos participantes ( $\mathrm{n}=471)$. Vale do Rio dos Sinos, ParanhanaEncosta da Serra e Hortênsias, RS, 2007-2011.

\begin{tabular}{lccccc}
\hline & \multicolumn{2}{c}{ Não } & \multicolumn{2}{c}{ Sim } \\
\hline \multicolumn{1}{c}{ Questão } & $\mathrm{n}$ & $\%$ & $\mathrm{n}$ & $\%$ \\
Você usa camisinha? & 397 & 84 & 58 & 12 \\
Você já realizou o teste anti-HIV? $^{2}$ & 428 & 91 & 38 & 8 \\
\hline
\end{tabular}

${ }^{1}$ Não informado $3 \%(15)$, nulo $0 \%(1) ;{ }^{2}$ não informado $1 \%(5)$.

Tabela 3 - Respostas dos participantes pré e pós-oficinas ( $\mathrm{n}=471)$. Vale do Rio dos Sinos, ParanhanaEncosta da Serra e Hortênsias, RS, 2007-2011.

\begin{tabular}{llccccc}
\hline \multicolumn{1}{c}{ Questão } & & \multicolumn{2}{c}{ Pré } & \multicolumn{2}{c}{ Pós } & $\mathrm{p}$ \\
\hline & Verdadeiro & 292 & 62 & 394 & $84 \%$ & 0,001 \\
& Falso & 10 & 2 & 15 & $3 \%$ & \\
O HIV é o causador da aids & Não sei & 153 & 33 & 54 & $11 \%$ & \\
& Não informado & 16 & 3 & 8 & $2 \%$ & \\
\hline \multirow{3}{*}{$\begin{array}{l}\text { A pessoa com o HIV sempre } \\
\text { apresenta os sintomas da }\end{array}$} & Verdadeiro & 184 & 39 & 141 & 30 & \\
doença & Falso & 72 & 15 & 218 & 46 & 0,001 \\
& Não sei & 201 & 43 & 108 & 23 & \\
& Não informado & 14 & 3 & 4 & 1 & \\
O HIV é identificado por meio & Verdadeiro & 380 & 80 & 420 & 89 & \\
de exames de laboratório & Falso & 9 & 2 & 13 & 3 & 0,001 \\
& Não sei & 74 & 16 & 35 & 7 & \\
& Não informado & 8 & 2 & 3 & 1 & \\
\hline \multirow{3}{*}{ O HIV pode ser transmitido } & Verdadeiro & 102 & 22 & 31 & 6 & 0,001 \\
por sabonetes, toalhas e & Falso & 238 & 50 & 367 & 78 & \\
assentos sanitários & Não sei & 126 & 27 & 70 & 15 & \\
& Não informado & 5 & 1 & 3 & 1 & \\
\hline
\end{tabular}




\begin{tabular}{|c|c|c|c|c|c|c|}
\hline \multicolumn{2}{|l|}{ Questão } & \multicolumn{2}{|c|}{ Pré } & \multicolumn{2}{|c|}{ Pós } & \multirow{2}{*}{$\frac{\mathrm{p}}{0,001}$} \\
\hline \multirow{4}{*}{$\begin{array}{l}\text { O HIV pode ser transmitido } \\
\text { por abraço, beijo no rosto, } \\
\text { beber no mesmo copo e } \\
\text { chimarrão }\end{array}$} & Verdadeiro & 85 & 18 & 28 & 6 & \\
\hline & Falso & 250 & 53 & 377 & 80 & \\
\hline & Não sei & 131 & 28 & 62 & 13 & \\
\hline & Não informado & 5 & 1 & 4 & 1 & \\
\hline \multirow{4}{*}{$\begin{array}{l}\text { O HIV pode ser transmitido } \\
\text { por picada de mosquito }\end{array}$} & Verdadeiro & 168 & 36 & 29 & 6 & 0,001 \\
\hline & Falso & 143 & 30 & 384 & 81 & \\
\hline & Não sei & 152 & 32 & 55 & 12 & \\
\hline & Não informado & 8 & 2 & 3 & 1 & \\
\hline \multirow{4}{*}{$\begin{array}{l}\text { A pessoa que usa camisinha } \\
\text { nas relações sexuais impede a } \\
\text { transmissão do HIV }\end{array}$} & Verdadeiro & 352 & 75 & 393 & 83 & 0,001 \\
\hline & Falso & 28 & 6 & 37 & 8 & \\
\hline & Não sei & 83 & 17 & 33 & 7 & \\
\hline & Não informado & 8 & 2 & 8 & 2 & \\
\hline \multirow{4}{*}{$\begin{array}{l}\text { Existe uma camisinha } \\
\text { específica para as mulheres }\end{array}$} & Verdadeiro & 300 & 64 & 432 & 92 & 0,001 \\
\hline & Falso & 13 & 2 & 12 & 2 & \\
\hline & Não sei & 155 & 33 & 24 & 5 & \\
\hline & Não informado & 3 & 1 & 3 & 1 & \\
\hline \multirow{4}{*}{$\begin{array}{l}\text { O uso da mesma seringa e } \\
\text { agulha por diversas pessoas } \\
\text { transmite o HIV }\end{array}$} & Verdadeiro & 423 & 90 & 391 & 83 & 0,001 \\
\hline & Falso & 14 & 2 & 53 & 10 & \\
\hline & Não sei & 32 & 7 & 26 & 6 & \\
\hline & Não informado & 2 & 1 & 1 & 1 & \\
\hline \multirow{4}{*}{$\begin{array}{l}\text { A aids é uma doença que ocorre } \\
\text { somente em homossexuais } \\
\text { masculinos, prostitutos(as) e } \\
\text { usuários(as) de drogas }\end{array}$} & Verdadeiro & 149 & 32 & 107 & 23 & 0,001 \\
\hline & Falso & 229 & 49 & 309 & 65 & \\
\hline & Não sei & 87 & 18 & 52 & 11 & \\
\hline & Não informado & 6 & 1 & 3 & 1 & \\
\hline \multirow{4}{*}{$\begin{array}{l}\text { Os indivíduos da terceira idade } \\
\text { não devem se preocupar com a } \\
\text { aids, pois ela atinge apenas os } \\
\text { jovens }\end{array}$} & Verdadeiro & 123 & 26 & 85 & 18 & 0,001 \\
\hline & Falso & 279 & 59 & 342 & 73 & \\
\hline & Não sei & 64 & 14 & 35 & 7 & \\
\hline & Não informado & 5 & 1 & 9 & 2 & \\
\hline \multirow{4}{*}{$\begin{array}{l}\text { A aids é uma doença que tem } \\
\text { tratamento }\end{array}$} & Verdadeiro & 352 & 75 & 424 & 90 & 0,001 \\
\hline & Falso & 39 & 8 & 19 & 4 & \\
\hline & Não sei & 72 & 15 & 21 & 4 & \\
\hline & Não informado & 8 & 2 & 7 & 2 & \\
\hline \multirow{4}{*}{$\begin{array}{l}\text { A aids é uma doença que tem } \\
\text { cura }\end{array}$} & Verdadeiro & 92 & 19 & 47 & 10 & 0,001 \\
\hline & Falso & 234 & 50 & 353 & 75 & \\
\hline & Não sei & 132 & 28 & 60 & 13 & \\
\hline & Não informado & 13 & 3 & 11 & 2 & \\
\hline
\end{tabular}

*O teste de McNemar foi utilizado para comparar as respostas pré e pós-oficinas. 
No domínio "conceito", a pergunta sobre a fase assintomática da infecção pelo HIV registrou a maior variação $(202,2 \%)$, segundo a tabela 4 .

Tabela 4 - Maiores variações obtidas em cada domínio pré e pós-oficinas. Vale do Rio dos Sinos, Paranhana-Encosta da Serra e Hortênsias, RS, 2007-2011.

\begin{tabular}{llccc}
\hline \multicolumn{1}{c}{ Domínio } & \multicolumn{1}{c}{ Questão } & Pré & Pós & $\%$ \\
\hline Conceito & $\begin{array}{l}\text { A pessoa com o HIV sempre apresenta os sintomas } \\
\text { da doença }\end{array}$ & 72 & 218 & 202,2 \\
Transmissão & O HIV pode ser transmitido por picada de mosquito & 143 & 384 & 168,53 \\
Prevenção & Existe uma camisinha específica para as mulheres & 300 & 432 & 44,00 \\
Vulnerabilidade & A aids é uma doença que ocorre somente em & 229 & 309 & 34,93 \\
& homossexuais masculinos, prostitutos(as) e & & & \\
Tratamento & usuários(as) de drogas & 234 & 353 & 50,85 \\
\hline
\end{tabular}

\section{DISCUSSÃO}

A maioria dos participantes desconhecia o período de latência clínica da infecção pelo HIV, denominado assintomático, no qual ocorre gradualmente a diminuição da resposta imunológica e que tem a duração média de três a sete anos. Durante a fase assintomática (assim como em qualquer etapa da infecção), o ciclo viral continua ativo e o indivíduo infectado pode transmitir o vírus para outras pessoas se não tomar medidas preventivas. ${ }^{11,12}$

O aumento significativo de acertos em todas as questões no domínio "transmissão" pode contribuir para a diminuição do estigma da doença e da discriminação das pessoas vivendo com HIV/Aids, sobretudo daquelas que estão inseridas na mesma comunidade dos indivíduos que participaram do presente estudo. ${ }^{13} \mathrm{Um}$ estudo com 3.600 pessoas de diversos estados brasileiros constatou maior nível de discriminação em pessoas de menor escolaridade, com faixa etária acima de 45 anos, e as mulheres apresentavam $66 \%$ a mais de chances de discriminação em relação aos homens. ${ }^{14}$
Considerando a mudança de perfil da epidemia, na qual a velocidade de crescimento é maior entre as mulheres do que entre os homens, a transmissão heterossexual é um fator importante a ser considerado na discriminação. Ou seja, o perfil das mulheres com HIV reitera que a prática de multiplicidade de parceiros é mais frequente e socialmente aceitável nos homens do que nas mulheres. ${ }^{15}$

$\mathrm{Na}$ questão que abordava o mosquito como um vetor na transmissão do HIV, ocorreu a segunda maior variação do estudo, representada por $168,53 \%$ (tabela 4). Nos estudos de Lazzarotto et al. ${ }^{10}$ e Fernandes ${ }^{16}$ foram encontrados os resultados de 41,4,\% e $41,1 \%$, respectivamente, para participantes que acreditavam que o mosquito poderia ser considerado um vetor da infecção. Na realização das oficinas, foi esclarecido que o mosquito não tem como ser um vetor para a transmissão do HIV. Além disso, a baixa infectividade e o curto período de sobrevivência do vírus no organismo do mosquito fazem com que esta forma de infecção seja inexistente. ${ }^{17} \mathrm{~A}$ literatura não relata nenhum caso dessa forma de transmissão. ${ }^{18}$ 
Considerando o exposto acima, no domínio "prevenção", as questões sobre o uso do preservativo e a existência de um preservativo para mulheres apresentaram aumentos significativos; mas quando questionados sobre o uso de preservativo nas relações sexuais, $87 \%$ dos participantes não o utilizavam. Tan et al., ${ }^{19}$ em estudo com homens acima de 50 anos em Singapura, encontraram 78 participantes que relataram ter intercurso sexual nos últimos seis meses com parceiros(as) não regulares (sexo casual ou com profissionais do sexo), sendo que 24,4\% não usaram preservativo. Dentre os 104 participantes desse estudo, ${ }^{19} 22,1 \%$ relataram que o preservativo afetava o desempenho sexual, e $73,1 \%$ afirmaram que reduzia o prazer sexual. Para Gir et al., ${ }^{20}$ o estado civil não é considerado um bom parâmetro para avaliação da atividade sexual e/ou o tipo de parceiro, visto que indivíduos com relacionamentos estáveis, por acreditarem em sua exclusividade em relação ao seu parceiro ou parceira sexual, não identificam seu companheiro como potencial risco para a infecção pelo HIV.

No processo de envelhecimento, as mulheres sofrem mudanças fisiológicas no trato genital, as paredes vaginais ficam mais finas e a lubrificação diminui, ficando mais fácil sofrer lesões durante as relações sexuais, o que aumentaria o risco de serem contaminadas pelo $\mathrm{HIV}^{7,12,19,21} \mathrm{O}$ estudo de Oliveira et al. ${ }^{22}$ salienta a importância da promoção e uso do preservativo feminino como forma de diminuir a desigualdade de sexo, por permitir às mulheres realizar o autocuidado quanto à saúde sexual, independentemente da opção do parceiro em relação à prevenção.

Para a concretização do autocuidado, fazem-se necessárias atividades educativas que promovam também a utilização deste insumo, proporcionando mais condições de autonomia às mulheres. Nesse contexto de prevenção, a distribuição de gel lubrificante junto com os preservativos masculino e feminino constituise uma necessidade que merece ser estudada pelo Ministério da Saúde, sobretudo para o público feminino, como justificativa para diminuir a incidência das infecções sexualmente transmissíveis, principalmente a aids, na população de idosos.

Políticas públicas que demonstrem a necessidade de uso do preservativo para esta faixa etária podem desmistificar a ideia de que o preservativo é utilizado apenas como método contraceptivo e salientar que pode prevenir a infecção pelo HIV, além de outras infecções sexualmente transmissíveis. ${ }^{7}$ Destaca-se também a importância de sensibilizar quanto ao problema, de modo a diminuir a resistência ao uso e minimizar os riscos. ${ }^{23}$

O estudo de Figueiredo \& Ayres $^{24}$ sobre intervenção realizada em mulheres de uma favela da cidade de São Paulo demonstrou que a associação de estratégias de distribuição de materiais educativos com a realização de grupos e a disponibilização de preservativo feminino e masculino são capazes de modificar a procura por prevenção de infecções sexualmente transmissíveis e aids, tendo em vista que a prevenção depende da sensibilização conjunta de homens e mulheres. Reitera-se que, durante as oficinas, eram apresentados os preservativos (masculinos e femininos) aos participantes com as devidas orientações sobre a utilização e no final eram distribuídos preservativos masculinos, fôlderes explicativos e cartazes. O preservativo feminino não foi distribuído devido a seu alto custo de aquisição no mercado.

A questão sobre o compartilhamento de seringas e agulhas por diversas pessoas poder transmitir o HIV foi a única que evidenciou diminuição no nível de conhecimento dos participantes $(p=0,001)$, conforme a tabela 3 . Contudo, alerta para o desenvolvimento de atitudes mais focalizadas na prevenção via parenteral. Tal diminuição pode ser atribuída ao fato de tanto homens como mulheres terem contato com instrumentos pérfuro-cortantes que, a priori, não estavam sendo considerados como meios de exposição ao HIV.

Ao abordar esse aspecto, houve dúvidas dos participantes e, como consequência, um 
número menor de respostas corretas após a oficina. Dentre as vias de transmissão do HIV, as agulhas contaminadas representam de 5 a $10 \%$ dos casos na epidemia mundial e ainda podem representar fator de risco na população acima de 40 anos de idade. ${ }^{12,25}$

No domínio "vulnerabilidade", questões em relação aos antigos e errôneos grupos de risco e a ideia de existir uma faixa etária mais vulnerável à infecção pelo HIV foram esclarecidas com a oficina. Alertou-se a faixa etária deste estudo para as situações de risco perante o HIV, como, por exemplo, o intercurso sexual desprotegido. A percepção de que a aids se limita a grupos específicos leva este grupo a determiná-la como doença fora do seu contexto, não sendo adotadas, assim, medidas preventivas. ${ }^{23,26}$

O "tratamento" foi o domínio em que se pôde modificar a informação errônea de que a aids é uma doença que tem cura, por meio de orientações aos participantes sobre a existência de tratamento, e que o mesmo está disponível nos Serviços de Assistência Especializada em HIV/Aids (SAEs). A realização da testagem periódica para HIV na meia-idade e velhice é muito importante, pois a história natural da infecção pelo HIV em pacientes mais velhos é diferente de pacientes mais jovens, ou seja, a imunossupressão é maior e a progressão da doença é mais rápida. ${ }^{12,13,27}$ Lekas et al..$^{28}$ sugerem a necessidade de intervenções que incentivem a redução das barreiras psicológicas para a testagem precoce para o HIV e a sensibilização para os riscos.

Houve redução das taxas mundiais da incidência do HIV/Aids, de 3,0 milhões em 2001 para 2,7 milhões em 2010. Segundo a Organização Mundial da Saúde (OMS), uma das explicações é o resultado dos programas de prevenção nos diferentes contextos, que propiciam a mudança de comportamento perante o HIV/Aids. Por esta razão, a OMS definiu que as campanhas sobre condutas sexuais seguras, generalização do acesso aos preservativos e combate à discriminação de pessoas que vivem com HIV/Aids, além das novas abordagens, como tratamento de infecções sexualmente transmissíveis, circuncisão masculina de forma voluntária e métodos para melhorar a retenção de pessoas que recebem terapia antirretroviral, servirão de estratégias para diminuir a disseminação do HIV e o impacto da aids. ${ }^{29-32}$

A entidade também ressalta que não pode definir corretamente o impacto de intervenções ou programas específicos, que acredita só ser possível por meio de estudos especiais em áreas específicas. ${ }^{30-32}$ Assim, confirma-se a importância de estudos como este, que definam a qualidade das intervenções realizadas em HIV/Aids, para que possam servir de referência qualificada aos programas de educação para a saúde que abranjam populações maiores de maneira mais efetiva. ${ }^{6,23}$

O estudo apresenta como limitações a ausência de um grupo controle, o cálculo do tamanho amostral e a aleatorização dos sujeitos em grupo controle e experimental, que proporcionariam maior controle sobre as variáveis e comparação intergrupos. Cabe acrescentar a inexistência de pesquisas que pudessem fortalecer a hipótese deste estudo: a realização de ações educativas sobre o HIV/Aids na população idosa aumenta o nível de conhecimento.

\section{CONCLUSÃO}

A realização das oficinas educativas sobre HIV/Aids demonstrou ser uma intervenção eficaz nos domínios "conceito", "transmissão", "prevenção", "vulnerabilidade" e "tratamento" nos participantes dos grupos de convivência das regiões do Vale do Rio dos Sinos, do ParanhanaEncosta da Serra e das Hortênsias-RS, Brasil. O acolhimento e o diálogo foram elementos essenciais para a construção de um ambiente profícuo de intervenção no contexto da terceira idade. Torna-se necessário que profissionais e gestores em saúde desenvolvam regularmente programas criativos de educação para a saúde. Almeja-se que os resultados deste estudo possam contribuir para diminuir a incidência de casos de aids na terceira idade e o preconceito com os portadores do HIV. 


\section{REFERÊNCIAS}

1. Brasil. Ministério da Saúde. Secretaria de Vigilância em Saúde. Departamento de Vigilância Epidemiológica. Doenças infecciosas e parasitárias: guia de bolso. 8. ed. Serie B. Textos Básicos de Saúde. Brasília: MS; 2010.

2. Araújo VLB, Brito DMS, Gimeniz MT, Queiroz TA, Tavares CM. Características da aids na terceira idade em um hospital de referência do Estado do Ceará, Brasil. Rev Bras Epidemiol 2007;10(4):544-54.

3. Brasil. Ministério da Saúde. Boletim Epidemiológico Aids e DST 2012. [acesso em 13 mar 2013]. Disponível em: http://www.aids.gov.br/publicacao/2012/boletimepidemiologico-aids-e-dst-2012 .

4. Veras R. Fórum. Envelhecimento populacional e as informações de saúde do PNAD: demandas e desafios contemporâneos. Cad Saúde Pública 2007;23(10):2463-6.

5. Rio Grande do Sul. Secretaria da Saúde. Avaliação da epidemia de aids no Rio Grande do Sul, dezembro 2007 [acesso em 12 jan 2012]. Disponível em: http:// www1.saude.rs.gov.br/dados/1217267856487Aids $\% 20$ RS\%20-\%20dez_2007\%20-\%20texto.pdf.

6. Lovejoy TI, Heckman TG, Sikkema KJ, Hansen NB, Kochman A, Suhr JA, et al. Patterns and correlates of sexual activity and condom use behavior in persons 50-plus years of age living with HIV/ AIDS. AIDS Behav 2008;12(6):943-56.

7. Bertoncini BZ, Moraes KS, Kulkamp IC. Comportamento sexual em adultos maiores de 50 anos infectados pelo HIV. DST - J Bras Doenças Sex Transm 2007;19(2):75-9.

8. Lazzarotto AR, Kramer AS, Hädrich M, Tonin M, Caputo P, Sprinz E. O conhecimento de HIV/aids na terceira idade: estudo epidemiológico no Vale do Sinos, Rio Grande do Sul, Brasil. Ciênc Saúde Coletiva 2008;13(6):1833-40.

9. Souza MHT, Backes DS, Pereira ADA, Ferreira CLL, Medeiros HMF, Marchiori MRCT. Nível de conhecimento de um grupo de idosos em relação à Síndrome da Imunodeficiência Adquirida. Av Enferm 2009;27(1):22-9.

10. Lazzarotto AR, Reichert MT, Venker C, Kramer AS, Sprinz E. HIV/aids e meia idade: avaliação do conhecimento de indivíduos da região do Vale do Sinos/RS, Brasil. Ciênc Saúde Coletiva 2010;15(1):1185-90.

11. Canini SRMS, Reis RB, Pereira LA, Gir E, Pelá NTR. Qualidade de vida de indivíduos com HIV/aids: uma revisão de literatura. Rev Latino-Am Enfermagem 2004;12(6):940-5.
12. Nguyen N, Holodniy M. HIV infection in the elderly. Clin Interv Aging 2008;3(3):453-72.

13. Brasileiro M, Freitas MI. Social representations about aids in people over 50, infected by HIV. Rev Latinoam Enfermagem 2006;14(5):789-95.

14. Garcia S, Koyama MAH; Grupo de Estudos em População, Sexualidade e Aids. Stigma, discrimination and HIV/AIDS in the Brazilian context, 1998 and 2005. Rev Saúde Pública 2008;42(Suppl 1):72-83.

15. Santos NJS, Barbosa RM, Pinho AA, Villela WV, Aidar T, Filipe EMV. Contextos de vulnerabilidade para o HIV entre mulheres brasileiras. Cad Saúde Pública 2009;25(Sup 2):S321-33.

16. Fernandes JCL. Evolução dos conhecimentos, atitudes e práticas relativas ao HIV/Aids em uma população de favela do Rio de Janeiro. Cad Saúde Pública 1998;14(3):575-81.

17. Iqbal MM. Can we get AIDS from mosquito bites? J La State Med Soc 1999;151(8):429-33.

18. Cohen MS, Hellmann N, Levy JA, DeCock K, Lange J. The spread, treatment, and prevention of HIV1: evolution of a global pandemic. J Clin Invest 2008;118(4):1244-54.

19. Tan HH, Wong ML, Chan RK. An epidemiological and knowledge, attitudes, beliefs and practices study of sexually transmitted infections in older men. Singapore Med J 2006;47(10):886-91.

20. Gir E, Canini SRMS, Carvalho MJ, Palos MAP. Reis RK, Duarte G . A parceria sexual na visão de mulheres portadoras do vírus da imunodeficiência humana - HIV. DST J Bras Doenças Sex Transm 2006;18(1):53-7.

21. Pogrányivá AH. El sida y las personas mayores. Gerokomos 2006;17(2):82-8.

22. Oliveira ICV, Araújo LF, Saldanha AAW. Percepções dos profissionais da saúde acerca da Aids na Velhice. DST J Bras Doenças Sex Transm 2006;18(2):143-7.

23. Zornitta M. Os novos idosos com aids: sexualidade e desigualdade à luz da bioética [tese de Mestrado]. Rio de Janeiro: Escola Nacional de Saúde Pública da Fiocruz; 2008.

24. Figueiredo R, Ayres JRCM. Intervenção comunitária e redução da vulnerabilidade de mulheres às DST/Aids em São Paulo, SP. Rev Saúde Pública 2002;36(4):96-107.

25. Caiaffa WT; Bastos FI. Usuários de drogas injetáveis e infecção pelo Vírus da Imunodeficiência Humana: epidemiologia e perspectivas de intervenção. Rev Bras Epidemiol 1998:1(2):190-202. 
26. Maia C, Guilhem D, Freitas D. Vulnerabilidade ao HIV/Aids de pessoas heterossexuais casadas ou em união estável. Rev Saúde Pública 2008;42(2):242-8.

27. Gebo KA. HIV and aging: implications for patient management. Drugs Aging 2006;23(11):897-913.

28. Lekas HM, Schrimshaw EW, Siegel K. Pathways to HIV testing among adults aged fifty and older with HIV/Aids. AIDS Care 2005;17(6):674-87.

29. World Health Organization. Towards universal access: scaling up priority HIV/AIDS interventions in the health sector. Geneva: WHO; 2010
30. Joint United Nations Programme on HIV/aids. Global report: UNAIDS report on the global AIDS epidemic 2010. Geneva: WHO; 2010.

31. Organización Mundial de la Salud. Estrategia mundial del sector sanitario para el VIH/SIDA, 2003-2007: un marco para las alianzas y la acción. Ginebra: OMS; 2003.

32. Organisation Mondiale de la Sante. Vers un accès universel : Étendre les interventions prioritaires liées au VIH/sida dans le secteur de la santé. Extraits du rapport de situation 2008. Genève: OMS; 2008. 